\title{
Downlink Spectral Efficiency of Distributed Antenna Systems Under a Stochastic Model
}

\author{
Yicheng Lin and Wei Yu
}

\begin{abstract}
This paper studies the downlink spectral efficiency of distributed antenna system (DAS) where antenna ports are distributed as a Poisson point process (PPP), while assuming channel state information is not available at the transmitter and each antenna has an individual power constraint. We first consider the case with a single user per cell and analyze regular DAS with fixed cell boundaries, and study both blanket transmission where the user is served by all the antenna ports within each cell, and selective transmission where only the closest antenna port to the user within each cell is selected. We derive efficiently computable spectral efficiency expressions as a function of the user location, and show the limitation of blanket transmission by establishing that the cell-edge spectral efficiency under blanket transmission is upper bounded by a constant. Further, from a network perspective, we also model users as a PPP and assume a TDMA-based user access, and give analytical expressions for and compare the average spectral efficiencies of regular DAS and user-centric $D A S$ where no fixed cell boundaries exist. We validate our models with simulation, and show that selective transmission outperforms blanket transmission for regular DAS, and usercentric DAS with selective transmission achieves a higher spectral efficiency averaged over the network than regular DAS.
\end{abstract}

Index Terms-Distributed antenna system, downlink spectral efficiency, multiple-input single-output (MISO), stochastic geometry, Poisson point process.

\section{INTRODUCTION}

$\mathbf{U}$ BIQUITOUS coverage and high data rates are key characteristics of future wireless systems. In traditional cellular systems, base-stations are located at the cell center to serve users which are spatially distributed across the cell. However, the dead spots within the cell caused by shadowing and penetration loss may significantly degrade the radio propagation strength of communication links, which in turn limit the system performance. Such performance degradation is most severe for the cell-edge users who suffer from both weaker signal strength due to relatively longer propagation distance and stronger inter-cell interference.

Distributed antenna system (DAS) provides a promising solution for improving coverage and capacity of wireless cellular networks [1]-[3]. Instead of deploying antennas centrally at the base-stations, by deploying remote antenna ports in coverage holes and connecting them with the home basestations via dedicated high-speed backhaul links, DAS can effectively mitigate detrimental effects such as shadowing and

The authors are with The Edward S. Rogers Sr. Department of Electrical and Computer Engineering, University of Toronto, 10 King's College Road, Toronto, Ontario M5S 3G4, Canada (e-mail: ylin@comm.utoronto.ca; weiyu@comm.utoronto.ca). This paper has been presented in part at the IEEE International Conference on Communications (ICC), Budapest, Hungary, June 2013. This work was supported by a Discovery Grant from the Natural Science and Engineering Research Council (NSERC) of Canada.

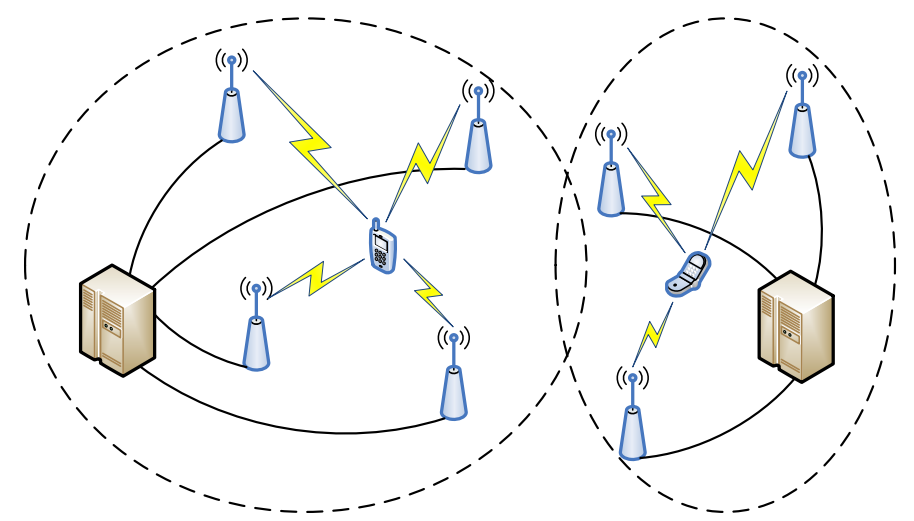

Fig. 1. An illustration of the distributed antenna system.

indoor penetration loss. The main advantage of DAS is that it statistically reduces the distance of a user to its nearest access point, thereby enhancing the received signal quality. In this way, the user performance in DAS is less sensitive to the user location within each cell. Fig. 1 shows an example of the DAS layout, where antenna ports are randomly deployed within each cell boundary to serve users.

This paper aims to provide a rigorous spectral efficiency analysis of downlink DAS under a stochastic model in which antennas are randomly distributed as a Poisson point process. We assume that channel state information (CSI) is not available at the transmitter and each antenna has an individual power constraint. For DAS with fixed cell boundaries, which we refer to as regular DAS, we consider two different transmission schemes: blanket transmission where all antenna ports within the cell cooperatively transmit to a given user, and selective transmission where the user is only served by the closest antenna port within the cell. The performance analysis of the present paper shows that selective transmission provides a higher spectral efficiency than blanket transmission under random antenna distribution. This confirms the empirical observation made in past literature, e.g., [4] for downlink and [5] for uplink. In addition, we consider a usercentric DAS architecture where the regular cellular structures no longer exist [5]. In particular, this paper analyzes selective transmission in this user-centric layout, and shows that it has a superior performance as compared to regular DAS from a network perspective. Note that this user-centric selective DAS model is analogous to the system model in [6].

In contrast to some previous studies on DAS which assume that antenna ports are deployed at fixed locations, e.g., uniformly on concentric circles as in [4], [7]-[9], in this paper 
we use a Poisson point process (PPP) to model the antenna locations. Such a random topology assumption is also adopted in other works on DAS [10]-[15]. The use of the PPP model is motivated by the fact that practical remote antennas are placed at arbitrary locations to cover the dead spots, so their locations can be modeled as a spatial random process from a birds-eye view of the network. It allows the use of theoretical results in stochastic geometry [16], [17] and provides tools for efficient numerical computation and analytical comparison of the spectral efficiencies of various DAS schemes.

A typical DAS architecture operates in the regime where the number of antennas exceeds the number of users in the system. We assume that CSI is not available at the transmitter. This assumption has been adopted in some of the DAS literatures, e.g., [4], [18], since it is often difficult to collect CSI for all the links in a system with a large number of distributed antennas. In the first part of this paper, we assume that each cell serves only one user, and analyze the location-specific spectral efficiency of such a system. In the second part of this paper, we assume that users are also randomly distributed in the network so that each cell may contain more than one users. In this case, we analyze the spectral efficiency of such a system from a network perspective where each cell serves multiple users in a time division multiple access (TDMA) manner in the absence of CSI.

\section{A. Related Work}

DAS is originally proposed to cover dead spots and improve coverage of wireless communication systems [1]. Benefits of DAS include reducing transmission power and outage probability, and most importantly, increasing capacity. The information theoretical capacity of DAS has been studied in some recent works. The authors of [4] suggest that DAS can reduce inter-cell interference and thus significantly improve capacity in a multi-cell environment, particularly for users near cell boundaries. They also show that selective diversity is better than blanket transmission in terms of capacity. The normalized achievable rate distributions of DAS under difference number of cooperative and interfering antennas are compared in [7], and an adaptive cooperation scheme is proposed. Under Rayleigh fading, the authors of [19] approximate the instantaneous DAS capacity to be Gaussian distributed, and the approximation is further extended in [18] for the DAS capacity under general fading and high signal-to-noise ratio (SNR) assumption. The idea of the virtual cell is introduced in [5], and the resulting user-centric DAS is analyzed for its outage performance in CDMA systems, where selective diversity is shown to outperform macrodiversity in the downlink. Most of the above studies either ignore the inter-cell interference or simply assume it to be Gaussian distributed. Explicitly modeled out-of-cell interference is included in [9], where the MIMO capacity of DAS under zero-forcing beamforming over all or a subset of antennas is evaluated.

The aforementioned studies of DAS all assume fixed and concentric deployment. The optimal antenna placement has been dealt with in several previous works, e.g., [8] develops a scheme based on the stochastic approximation theory, and [20] proposes a placement optimization for single-cell and twocell systems by maximizing the lower bound of either SNR or the signal-to-leakage ratio (SLR). However, in this paper the placement of antennas is not an optimization objective. Instead, we model the antennas as a PPP to capture the spatial randomness in practice.

The analysis of wireless systems under random topology has been carried out for cellular networks [21], [22] and heterogeneous networks [23]-[25] in the literatures. For DAS, the randomness of the node locations has been considered in [10]-[12] for downlink and in [13]-[15] for uplink. The advantage of DAS over centralized system is shown in [10] by comparing the statistics of mean square access distance. In [11], the authors focus on the outage probability of DAS by approximating the out-of-cell interference as a single lognormal random variable. Using random matrix theory, [12] gives an approximation of the DAS capacity, where large-scale fading is assumed to be static and interference is assumed to be Gaussian distributed. In [13], the authors use the mean square access distance as a performance metric for the uplink system, assuming interference from other users is eliminated, and reveal that given a fixed total number of antennas, it is better to deploy more antenna clusters with fewer antennas per cluster. The comparative study in [14] shows that decentralized systems yield a much higher capacity than traditional colocated systems. In [15], using the central limit theorem, the uplink mutual information is approximated to be Gaussian and log-normal distributed at high and low SNR, respectively, and the transmission efficiencies of both DAS and co-located MIMO are compared under different antenna deployments.

Different from the aforementioned works on DAS with random antenna deployment in the downlink [10]-[12], this paper characterizes the spectral efficiency of DAS (instead of other metrics such as mean square access distance [10] or outage probability [11]), and explicitly models the desired signal as well as the inter-cell/cluster interference (instead of modeling interference as a Gaussian random variable [12]). We use tools from stochastic geometry to analyze and compare different DAS schemes.

\section{B. Main Results}

This paper studies the downlink spectral efficiency of DAS with random antenna locations. We analyze regular DAS with blanket and selective transmission, as well as usercentric selective DAS. The traditional cellular system where all antennas are co-located at the cell center is also presented as a reference. Using stochastic geometry, we derive spectral efficiency formulas for DAS which enable fast numerical computation without the need to resort to Monte Carlo simulations. Our analytical model matches simulations of different DAS schemes for most cases. Specifically,

- For regular DAS, we characterize the location-specific spectral efficiency of a given user, i.e., spectral efficiency as a function of the distance from the user to the cell center, for both blanket and selective transmission.

- Using these expressions, we show that the cell-edge spectral efficiency of regular DAS with blanket transmission 
is upper bounded by $\frac{\alpha}{2}$ bits $/ \mathrm{s} / \mathrm{Hz}$, which is a function of the path loss exponent $\alpha$ only and is independent of the antenna port intensity or the cell radius; while no such bound is found for the selective scheme.

- Assuming that users are also PPP modeled, we analyze and compare the average spectral efficiencies of regular and user-centric DAS from a network perspective.

These derived expressions facilitate a comprehensive numerical comparison of different DAS layouts and transmission schemes. Based on the numerical results, we conclude for the downlink DAS with random topology as follows.

- Fully distributed DAS (one antenna per port) outperforms partially distributed DAS (multiple antennas grouped at a port) with the same total number of antennas. This is in accordance with the conclusion in [13] for uplink DAS.

- Regular DAS achieves a higher spectral efficiency than the traditional cellular system for users at cell edges. With fixed antenna locations, [4] reveals the same conclusion.

- For regular DAS, selective transmission achieves a higher spectral efficiency as compared to blanket transmission. Similar observations are made in [4] for downlink DAS with fixed locations and [5] for uplink DAS.

- User-centric selective DAS achieves a higher spectral efficiency than both regular DAS schemes when averaged over the network.

\section{Organization of the Paper}

Section II describes the system model and establishes the general DAS spectral efficiency expression under the MISO channel. Section III analyzes the spectral efficiency of a single user at a specified location within the cell for regular DAS with both blanket and selective transmission, and presents numerical results. In Section IV, the average spectral efficiency of a randomly chosen user is studied for both regular and usercentric DAS, and the comparison of all schemes are given. Section V draws conclusions.

\section{DAS SPeCtral EFFICIENCY UNDER MISO ChanNEL}

This section derives a general spectral efficiency formula for DAS with a single user per cell. The user is assumed to be equipped with a single antenna, and the link from the distributed antennas within the cell to the user is modeled as a multiple-input single-output (MISO) channel. We assume each individual antenna, no matter co-located at a port or individually placed, has a separate power constraint. This is justified by the fact that in practical implementation each antenna is equipped with its own power amplifier and the transmission power at the antenna is limited individually by the linearity range of the amplifier. Without transmitter-side CSI and with per-antenna power constraint, the spectral efficiency computed in this paper for this setting is in fact the multipleaccess channel capacity, which also equals the ergodic MISO channel capacity under the same CSI and power assumption [26]. With Gaussian signaling, the general form of the spectral efficiency (in nats $/ \mathrm{s} / \mathrm{Hz}$ ) of the user in cell $o$ (or cluster $o$ ) under the DAS model is

$$
\mathcal{C}=\mathbb{E}_{\mathbf{h}}\left[\ln \left(1+\frac{\sum_{n} P_{n}^{(o)}\left|h_{n}^{(o)}\right|^{2}\left\{r_{n}^{(o)}\right\}^{-\alpha}}{\sum_{w \neq o} \sum_{m} P_{m}^{(w)}\left|h_{m}^{(w)}\right|^{2}\left\{r_{m}^{(w)}\right\}^{-\alpha}+\sigma^{2}}\right)\right],
$$

where $n$ and $m$ are the antenna indices, $o$ and $w$ are the cell (cluster) indices, $P_{n}^{(o)}$ is the transmit power of antenna $n$ in cell (cluster) $o$ with a fixed value $\mu, h_{n}^{(o)} \sim \mathcal{C N}(0,1)$ is the complex channel coefficient with Rayleigh distributed amplitude from antenna $n$ in cell (cluster) $o$ to the considered user, $\left\{r_{n}^{(o)}\right\}^{-\alpha}$ models the path loss where $\alpha$ is the path loss exponent (typically $\alpha>2$ ), and $\sigma^{2}$ is the noise power. We consider the case where only one user is served in each cell (cluster) at each time/frequency unit, thus (1) is also the spectral efficiency per cell (cluster). Note that with selective transmission, $o$ and $w$ become the indices of the antenna ports that are actively serving users.

The above expectation is averaged over the channel $\mathbf{h}$ and does not take the random antenna port locations into account. We now further assume that the distributed antenna ports can be modeled as a homogeneous PPP $\Phi_{A}$, and denote $\Phi_{A}^{(S)}$ and $\Phi_{A}^{(I)}$ as the subsets of points over $\mathbb{R}^{2}$ that consist of, respectively, antenna ports in the serving cell (cluster) $o$ and antenna ports in all the interfering cells (clusters) $w, w \neq o$. For the general partially distributed case, we assume that every $K$ of the antennas are co-located within one antenna port. Let $i$ and $j$ be the antenna port indices, $p$ and $q$ be the antenna indices within one port. We use $P_{i p}$ and $h_{i p}$ to denote the power and the Rayleigh fading of antenna $p$ in port $i$, and $r_{i}$ is its distance to the user. Note that the antennas at the same port have the same path loss attenuation. For notational simplicity we denote

$$
G_{i}=\sum_{p=1}^{K} P_{i p}\left|h_{i p}\right|^{2}=\mu \sum_{p=1}^{K}\left|h_{i p}\right|^{2}, \quad \forall i .
$$

Let $A_{i}$ be the location of antenna port $i$, we can now rewrite (1) by dropping the cell (cluster) index $o$ and $w$ as

$$
\mathcal{C}=\mathbb{E}_{\Phi_{A}, \mathbf{G}}\left[\ln \left(1+\frac{\sum_{A_{i} \in \Phi_{A}^{(S)}} G_{i} r_{i}^{-\alpha}}{\sum_{A_{j} \in \Phi_{A}^{(I)}} G_{j} r_{j}^{-\alpha}+\sigma^{2}}\right)\right],
$$

where the expectation is taken over both the PPP $\Phi_{A}$ and the equivalent channel $\mathbf{G}$.

The following lemma is useful in further deriving the spectral efficiency expressions of different DAS schemes.

Lemma 1: The DAS downlink spectral efficiency under the PPP antenna model can be expressed as

$$
\mathcal{C}\left(\mathcal{G}_{\Phi_{A}^{(S)}}, \mathcal{G}_{\Phi_{A}^{(I)}}\right)=\int_{0}^{\infty} \frac{e^{-s \sigma^{2}}}{s} \mathcal{G}_{\Phi_{A}^{(I)}}\left(1-\mathcal{G}_{\Phi_{A}^{(S)}}\right) \mathrm{d} s
$$

where $\mathcal{G}_{\Phi_{A}^{(S)}}$ and $\mathcal{G}_{\Phi_{A}^{(I)}}$ are the Laplace transforms of the 
desired signal and the interference parts, respectively,

$$
\begin{aligned}
& \mathcal{G}_{\Phi_{A}^{(S)}}=\mathbb{E}_{\Phi_{A}, \mathbf{G}}\left(\prod_{A_{j} \in \Phi_{A}^{(S)}} e^{-s G_{j} r_{j}^{-\alpha}}\right) \\
& \mathcal{G}_{\Phi_{A}^{(I)}}=\mathbb{E}_{\Phi_{A}, \mathbf{G}}\left(\prod_{A_{j} \in \Phi_{A}^{(I)}} e^{-s G_{j} r_{j}^{-\alpha}}\right)
\end{aligned}
$$

Proof: From equation (3),

$$
\begin{aligned}
\mathcal{C} \stackrel{(a)}{=} \mathbb{E}_{\Phi_{A}, \mathbf{G}}\left\{\int_{0}^{\infty} \frac{e^{-z}}{z}\left[1-\exp \left(\frac{-z \sum_{A_{i} \in \Phi_{A}^{(S)}} G_{i} r_{i}^{-\alpha}}{\sum_{A_{j} \in \Phi_{A}^{(I)}} G_{j} r_{j}^{-\alpha}+\sigma^{2}}\right)\right] \mathrm{d} z\right\} \\
\stackrel{(b)}{=} \mathbb{E}_{\Phi_{A}, \mathbf{G}}\left\{\int_{0}^{\infty} \frac{e^{-s \sigma^{2}}}{s} \exp \left(-s \sum_{A_{j} \in \Phi_{A}^{(I)}} G_{j} r_{j}^{-\alpha}\right)\right. \\
\stackrel{(c)}{=} \int_{0}^{\infty} \frac{e^{-s \sigma^{2}}}{s} \mathbb{E}_{\Phi_{A}, \mathbf{G}}\left(\prod_{A_{j} \in \Phi_{A}^{(I)}} e^{-s G_{j} r_{j}^{-\alpha}}\right) \\
\left.\times\left[1-\exp \left(\begin{array}{c}
-s \sum_{A_{i} \in \Phi_{A}^{(S)}} G_{i} r_{i}^{-\alpha} \\
-
\end{array}\right)\right] \mathrm{d} s\right\} \\
{\left[\mathbb{E}_{\Phi_{A}, \mathbf{G}}\left(\prod_{A_{i} \in \Phi_{A}^{(S)}} e^{-s G_{i} r_{i}^{-\alpha}}\right)\right] \mathrm{d} s }
\end{aligned}
$$

where $(a)$ follows from the Lemma 1 in [27]:

$$
\ln (1+x)=\int_{0}^{\infty} \frac{e^{-z}}{z}\left(1-e^{-x z}\right) \mathrm{d} z,
$$

and (b) follows from a change of variable $z=$ $s\left(\sum_{j \in \Phi_{A}^{(I)}} G_{j} r_{j}^{-\alpha}+\sigma^{2}\right)$. The expectation and integration are interchanged in $(c)$ by applying the Fubini theorem (since the integrand is non-negative), and by recognizing that the subsets $\Phi_{A}^{(S)}$ and $\Phi_{A}^{(I)}$ are disjoint. By plugging in $\mathcal{G}_{\Phi_{A}^{(S)}}$ and $\mathcal{G}_{\Phi_{A}^{(S)}}$ we have (4).

\section{Spectral EfFiciency of Regular DAS FROM A LoCATION-SPECIFIC PerspeCtive}

This section considers DAS in the conventional sense where the coverage area of each cell is defined by the fixed cell boundary. This deployment topology is called the regular DAS layout in this paper. We provide the spectral efficiency analysis for regular DAS from a location-specific perspective, assuming that there is a single user in the cell. The location here refers to the distance between the specific user and the cell center.

Different from [21], [22] where all cells are derived from Voronoi tessellation [28], we adopt a hybrid approach as in [29]: the center serving cell is of fixed size and modeled as a circle with radius $R$, while the shape of interfering cells can be arbitrary. Thus $\Phi_{A}^{(S)}$ and $\Phi_{A}^{(I)}$ are sets of antenna ports within and outside of the cell radius $R$, respectively, as in Fig. 2 .

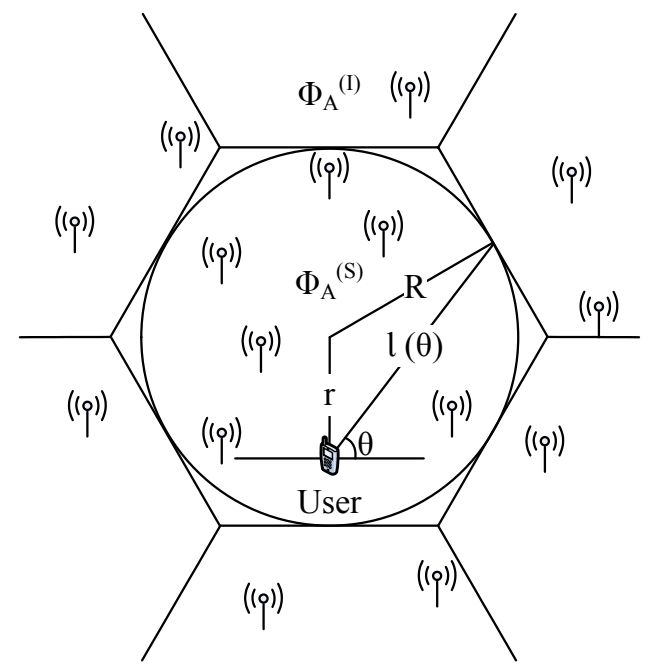

Fig. 2. The regular DAS layout. The boundary of the cell under consideration is modeled as a circle, and the boundaries of interfering cells can be arbitrary.

Let the average number of antennas per cell be $N$. Assuming exactly $K$ antennas per port, the average number of antenna ports per cell is therefore $\frac{N}{K}$. We assume that the average size of the interfering cells is $\pi R^{2}$, i.e., the same as the serving cell. In this section, we make a key assumption that there is exactly one user being served at a time in each cell, and compute the spectral efficiency for that user as a function of its location. This assumption will be removed and the analysis will be extended in the next section to provide a network-wide spectral efficiency characterization for DAS.

\section{A. Blanket Transmission}

Blanket transmission refers to the case in which all antennas of each cell form a macrodiversity group by transmitting to one scheduled user which is randomly placed within the cell. The spatial intensity of the antenna ports in $\Phi_{A}^{(S)}$ is therefore $\lambda_{A}^{(S)}=\frac{N}{K \pi R^{2}}$. Assuming homogeneity, we have $\lambda_{A}=\lambda_{A}^{(S)}=$ $\lambda_{A}^{(I)}$, where $\lambda_{A}^{(I)}$ is the intensity of the set $\Phi_{A}^{(I)}$.

Setting the location of the considered user in the cell $o$ as the origin, we have the following theorem.

Theorem 1: For regular DAS with blanket transmission, the downlink spectral efficiency of the user with distance $r \leq R$ from the cell center is

$$
\begin{aligned}
\mathcal{C}_{r}^{(\text {Blk. })}= & \int_{0}^{\infty}\left\{\exp \left[\frac{1}{2} \lambda_{A}(\mu s)^{2 / \alpha} \int_{0}^{2 \pi} \int_{\ell^{2}(\theta)(\mu s)^{-2 / \alpha}}^{\infty} \beta(u) \mathrm{d} u \mathrm{~d} \theta\right]\right. \\
& \left.-\exp \left[\pi \lambda_{A}(\mu s)^{2 / \alpha} \int_{0}^{\infty} \beta(u) \mathrm{d} u\right]\right\} \frac{e^{-s \sigma^{2}}}{s} \mathrm{~d} s
\end{aligned}
$$

where $\beta(u)<0$ has the form of

$$
\beta(u)=\left(1+u^{-\alpha / 2}\right)^{-K}-1 .
$$

The term $\ell(\theta)$ in (8) is the distance from the user to the cell edge at the angle $\theta$, which is also a function of the distance $r$ and the cell radius $R$, as shown in Fig. 2. Specifically,

$$
\ell(\theta)=\sqrt{R^{2}-r^{2} \cos ^{2} \theta}+r \sin \theta .
$$


Proof: We start from (4) to get the Laplace transforms of the desired signal and interference parts. For the signal part

$$
\begin{aligned}
\mathcal{G}_{\Phi_{A}^{(S)}}^{(\text {Blk. })} & \stackrel{(a)}{=} \mathbb{E}_{\Phi_{A}}\left[\prod_{A_{i} \in \Phi_{A}^{(S)}} \mathbb{E}_{\mathbf{G}}\left(e^{-s G_{i} r_{i}^{-\alpha}}\right)\right] \\
& \stackrel{(b)}{=} \mathbb{E}_{\Phi_{A}}\left[\prod_{A_{i} \in \Phi_{A}^{(S)}}\left(1+\mu s r_{i}^{-\alpha}\right)^{-K}\right] \\
& \stackrel{(c)}{=} \exp \left\{\lambda_{A} \int_{0}^{2 \pi} \int_{0}^{\ell(\theta)}\left[\left(1+\mu s v^{-\alpha}\right)^{-K}-1\right] v \mathrm{~d} v \mathrm{~d} \theta\right\} .
\end{aligned}
$$

In $(a)$ we use the i.i.d. property of the fading channels among antenna ports and their independence from the PPP $\Phi_{A}$. Assuming independence of the fading among antennas of one port in $(b)$, we have $G_{i} \sim \Gamma(K, \mu)$, which simplifies to $G_{i} \sim \exp \left(\frac{1}{\mu}\right)$ when $K=1$. Step $(c)$ follows from the probability generating functional (p.g.fl.) [17] of a PPP $\Phi$ with intensity $\lambda(x)$ such that

$$
\mathbb{E}_{\Phi}\left[\prod_{x \in \Phi} f(x)\right]=\exp \left\{\int_{\mathbb{R}^{2}}[f(x)-1] \lambda(x) \mathrm{d} x\right\},
$$

and a conversion from Cartesian to polar coordinates.

Similarly, for the interference part in (4) we have

$$
\mathcal{G}_{\Phi_{A}^{(I)}}^{(\mathrm{Blk})}=\exp \left\{\lambda_{A} \int_{0}^{2 \pi} \int_{\ell(\theta)}^{\infty}\left[\left(1+\mu s v^{-\alpha}\right)^{-K}-1\right] v \mathrm{~d} v \mathrm{~d} \theta\right\} .
$$

By employing a change of variables $u=v^{2}(\mu s)^{-2 / \alpha}$ in (11) and (13) and plugging them back to (4), after some manipulations we have (8).

The spectral efficiency expression (8) can be further simplified if we consider the special case of $K=1$ (corresponding to the fully distributed antenna deployment), $\alpha=4, \sigma^{2}=0$ (thus the power has no influence on the spectral efficiency and we can use $\mu=1$ ). In this case,

$$
\mathcal{C}_{r}^{(\text {Blk. })}=\int_{0}^{\infty} \frac{1}{s} e^{-\frac{1}{2} \pi^{2} \lambda_{A} s^{1 / 2}}\left(e^{\frac{1}{2} \lambda_{A} s^{1 / 2} \tau(s)}-1\right) \mathrm{d} s,
$$

where

$$
\tau(s)=\int_{0}^{2 \pi} \arctan \left[\ell^{2}(\theta) s^{-1 / 2}\right] \mathrm{d} \theta .
$$

Fixing $\lambda_{A}$ and $r$, the spectral efficiency (8) is an increasing function of $R$, since $\ell(\theta)$ increases with $R$ for all $\theta$ and $\beta(u)<$ 0 . (Note that in this case $\frac{N}{K}$ grows as $\mathcal{O}\left(R^{2}\right)$.) In addition, the spectral efficiency at the cell edge is also an increasing function of $R$ (i.e., when $r=R$ ). This gives the following spectral efficiency upper bound for the cell-edge user:

Corollary 1: For regular DAS, the downlink cell-edge spectral efficiency is upper bounded by $\frac{\alpha}{2}$ bits $/ \mathrm{s} / \mathrm{Hz}$.

Proof: Since spectral efficiency increases with $R$, we only need to consider the limiting case $r=R=\infty$, where $\ell(\theta)$ is

$$
\ell(\theta)_{\mid r=R=\infty}= \begin{cases}2 R \sin \theta_{\mid R=\infty}=\infty & \theta \in[0, \pi) \\ 0 & \theta \in[\pi, 2 \pi) .\end{cases}
$$

Substituting (16) into (8) and ignoring the noise (i.e., $\sigma^{2}=0$ ), after some manipulations, the upper bound on the cell-edge spectral efficiency can be derived as

$$
\begin{aligned}
\mathcal{C}_{r=R}^{(\text {Blk. })} & <\mathcal{C}_{r=R=\infty}^{\text {(Blk.) }} \\
& =\int_{0}^{\infty} \frac{1}{s}\left(e^{-Q s^{2 / \alpha}}-e^{-2 Q s^{2 / \alpha}}\right) \mathrm{d} s \\
& \stackrel{(a)}{=} \frac{\alpha}{2} \int_{0}^{\infty} \frac{e^{-Q t}}{t}\left(1-e^{-Q t}\right) \mathrm{d} t \\
& \stackrel{(b)}{=} \frac{\alpha}{2} \ln (1+1) \\
& =\frac{\alpha}{2} \ln (2) \text { nats/s/Hz } \\
& =\frac{\alpha}{2} \text { bits/s/Hz, }
\end{aligned}
$$

where $Q=\frac{1}{2} \pi \lambda_{A} \mu^{2 / \alpha} \int_{0}^{\infty} \beta(u) \mathrm{d} u,(a)$ follows from a change of variables $t=s^{2 / \alpha}$, and (b) follows from equation (7).

This corollary shows that although DAS is expected to have a better coverage than the traditional cellular system at the cell edge, the cell-edge spectral efficiency of regular DAS with blanket transmission is still bounded by a constant, which is solely determined by the path loss exponent and is independent of the antenna port intensity. This is a severe limitation of the blanket transmission scheme of DAS architecture.

This upper bound is derived by letting the cell size go to infinity. In such a limit, the cell boundary is locally a straight line, with the serving and the interfering antenna ports distributed on each half-plane with the same intensity.

\section{B. Selective Transmission}

Selective transmission in DAS refers to the transmission strategy in which only the antenna port closest to the user is selected to transmit within each cell as in [4], [11]. Note that it is possible to generalize this selective transmission scheme to a $k$-selective scheme, in which the user communicates with $k$ closest antenna ports. This generalized approach is however more difficult to analyze. The analysis in this paper is restricted to the case of $k=1$.

Under the PPP assumption of the antenna ports, there are occasions when a cell has no antennas, and we denote void as the event of having at least one antenna within a cell, which has the probability

$$
\mathbb{P}_{A}(\overline{\text { void }})=1-e^{-\pi R^{2} \lambda_{A}} .
$$

To utilize (4), we again need to obtain the Laplace transforms of the signal and interference parts. Conditioned on the existence of antennas in the considered cell, the signal set $\Phi_{A}^{(S)}$ contains one selected antenna port. The signal part hence simplifies to

$$
\begin{aligned}
\mathcal{G}_{\Phi_{A}^{(S)} \mid \overline{\text { void }}}^{(\text {Sel })} & =\mathbb{E}_{d, G}\left(e^{-s G d^{-\alpha}}\right)=\mathbb{E}_{d}\left[\mathbb{E}_{G}\left(e^{-s G d^{-\alpha}}\right)\right] \\
& =\mathbb{E}_{d}\left[\left(1+\mu s d^{-\alpha}\right)^{-K}\right]
\end{aligned}
$$

where $d$ is the antenna-to-user distance within the cell, for which we arrive at the following lemma:

Lemma 2: For the regular DAS layout, conditioned on having at least one antenna within the serving cell, the distribution of the distance between the considered user located 


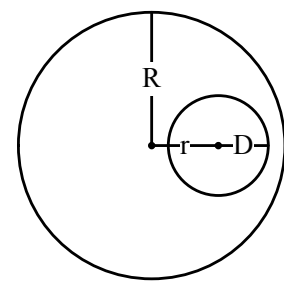

(a) $D \leq R-r$

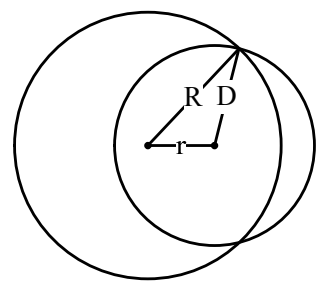

(b) $R-r<D \leq R+r$
Fig. 3. Intersection area of the cell and user disc with radius $D$.

at a distance $r \leq R$ away from the cell center and its closest antenna port within the cell boundary is

$f_{d}(d \mid \overline{\text { void }})= \begin{cases}\frac{2 \pi d \lambda_{A}}{1-\exp \left(-\pi R^{2} \lambda_{A}\right)} e^{-\pi d^{2} \lambda_{A}} & d \in[0, R-r] \\ \frac{2 d \lambda_{A}}{1-\exp \left(-\pi R^{2} \lambda_{A}\right)} e^{-\mathcal{X}(R, r, d) \lambda_{A}} \\ \times \arccos \left(\frac{r^{2}-R^{2}+d^{2}}{2 r d}\right) & d \in(R-r, R+r] \\ 0 & d \in(R+r,+\infty) .\end{cases}$

where

$$
\begin{aligned}
& \mathcal{X}(R, r, d)= \\
& d^{2} \arccos \left(\frac{r^{2}-R^{2}+d^{2}}{2 r d}\right)+R^{2} \arccos \left(\frac{r^{2}+R^{2}-d^{2}}{2 r R}\right) \\
& -\frac{1}{2} \sqrt{(r+R+d)(r-R+d)(r+R-d)(-r+R+d)} .
\end{aligned}
$$

Proof: We start by finding the conditional probability $\mathbb{P}(d>D \mid \overline{\text { void }})$, i.e., the probability that no antennas within the cell boundary are closer to the considered user than a distance $D$. Equivalently, this is the null probability of PPP in the intersection area of the cell and the disc with radius $D$ centered at the user. This intersection area is a function of the radii $R$ and $D$ of the two circles as well as the distance $r$ between their centers. As illustrated in Fig. 3, the area is $\pi D^{2}$ if $D \leq R-r$ and is $\mathcal{X}(R, r, D)$ as in (21) if $R-r<D \leq R+r$. Thus we have

$$
\begin{aligned}
& \mathbb{P}(d>D \mid \overline{\text { void }})=\frac{\mathbb{P}(d>D)}{\mathbb{P}(\overline{\text { void }})}=\frac{\mathbb{P}(d>D)}{1-e^{-\pi R^{2} \lambda_{A}}} \\
& = \begin{cases}\frac{1}{1-\exp \left(-\pi R^{2} \lambda_{A}\right)} e^{-\pi D^{2} \lambda_{A}} & D \in[0, R-r] \\
\frac{1}{1-\exp \left(-\pi R^{2} \lambda_{A}\right)} e^{-\mathcal{X}(R, r, D) \lambda_{A}} & D \in(R-r, R+r] \\
0 & D \in(R+r,+\infty) .\end{cases}
\end{aligned}
$$

Consequently $f_{d}(d \mid \overline{\text { void }})=\frac{\mathrm{d}[1-\mathbb{P}(d>D \mid \overline{\text { void }})]}{\mathrm{d} D} \mid D=d$ as in (20).

Based on Lemma 2, the signal part is now

$$
\mathcal{G}_{\Phi_{A}^{(S)} \mid \overline{\text { void }}}^{(\text {Sel. }}=\int_{0}^{R+r}\left(1+\mu s d^{-\alpha}\right)^{-K} f_{d}(d \mid \overline{\text { void }}) \mathrm{d} d \text {. }
$$

To derive the corresponding characterization of the interference part, we note that in each interfering cell one antenna port is selected to serve one user. Since the locations of the selected antenna ports are arbitrary, the set of interfering antenna ports
$\Phi_{A}^{(I)}$ can be well approximated as a PPP. Considering that a cell with no antennas does not generate interference, the intensity of this PPP is a thinned version of the intensity of the interfering cells (given the average cell size $\pi R^{2}$ )

$$
\lambda_{A}^{(\text {Sel. })}=\mathbb{P}_{A}(\overline{\text { void }}) \frac{1}{\pi R^{2}}=\left(1-e^{-\pi R^{2} \lambda_{A}}\right) \frac{1}{\pi R^{2}} .
$$

Hence following the similar procedures as in (13) we have

$$
\mathcal{G}_{\Phi_{A}^{(I)}}^{(\text {Sel. })} \approx \exp \left[\frac{1}{2} \lambda_{A}^{(\text {Sel. })}(\mu s)^{2 / \alpha} \int_{0}^{2 \pi} \int_{\ell^{2}(\theta)(\mu s)^{-2 / \alpha}}^{\infty} \beta(u) \mathrm{d} u \mathrm{~d} \theta\right]_{(25)}
$$

Similar to (6) and considering the zero-antenna event we have the following proposition.

Proposition 1: For regular DAS with selective transmission, the downlink spectral efficiency of the user with distance $r \leq$ $R$ from the cell center is approximated by

$$
\mathcal{C}_{r}^{(\text {Sel. })}=\mathbb{P}_{A}(\overline{\text { void }}) \mathcal{C}_{r \mid \text { void }}^{\text {(Sel. }}
$$

where

$$
\mathcal{C}_{r \mid \text { void }}^{(\text {Sel. })}=\int_{0}^{\infty} \frac{e^{-s \sigma^{2}}}{s} \mathcal{G}_{\Phi_{A}^{(I)}}^{(\text {Sel. })}\left(1-\mathcal{G}_{\Phi_{A}^{(S)} \mid \text { void }}^{(\text {Sel. }}\right) \mathrm{d} s,
$$

and $\mathbb{P}_{A}(\overline{\text { void }}), \mathcal{G}_{\Phi^{(I)}}^{(\text {Sel. })}$ and $\mathcal{G}_{\Phi_{A}^{(S)} \mid \overline{\text { void }}}^{(\text {Sel. })}$ are given in (18), (23), and (25), respectively.

\section{Comparison of Blanket and Selective Transmission}

We present numerical simulations to verify our theoretical results, and compare the two regular DAS schemes in the location-specific analysis. Recall that $N$ is the average antenna number per cell and $K$ is the antenna number per port. We assume that the per-antenna power is $\frac{P}{N}$, where $P=46 \mathrm{dBm}$. We set the cell radius to $R=1000 \mathrm{~m}$ and vary the userto-cell-center distance $r$. Compared with the Monte Carlo experiments, the analytical integrations can be computed more efficiently, and its effectiveness is verified with simulations as in Figs. 4-7.

Note that spectral efficiency drops from the cell center to the cell edge as expected. Also, with a stochastic-geometry-based analysis there is no such "rise-and-drop" effect for spectral efficiency as observed in [4], [9] for fixed deployment (i.e., spectral efficiency rises as the user gets closer to a antenna port at a fixed location, and drops when it is farther away). This is because in a stochastic DAS topology antenna ports are not fixed and are distributed randomly according to a spatial point process. The simulation results for the regular cellular topology are depicted as "Grid Cellular" in Fig. 4, where all $N$ antennas are co-located at each cell center, and all cell centers are regularly placed to form a hexagonal layout. As compared to the centralized cellular architecture, the spectral efficiency of DAS is higher at the cell edge but lower at the cell center, which illustrates DAS's ability in achieving ubiquitous coverage. Another observation from Fig. 4 is that the gap between $N=1$ and $N=24$ is much more significant for DAS than for the co-located cellular system. This is because unlike the latter layout, increasing the total antenna number in DAS statistically shortens the distance between a user and its 

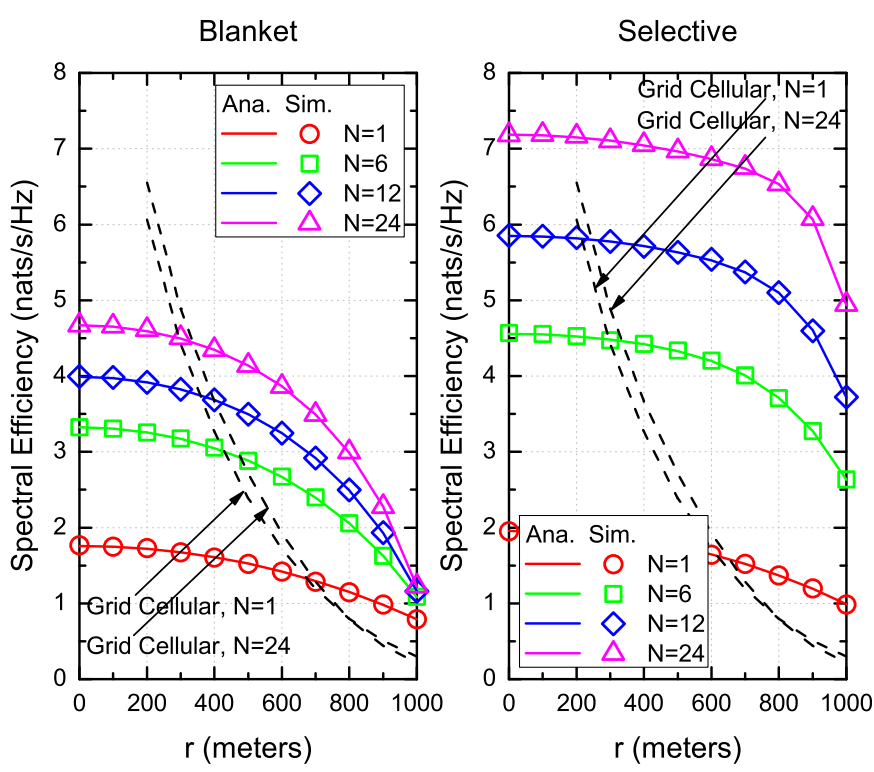

Fig. 4. Grid cellular vs. regular DAS: spectral efficiency as a function of the cell-center-to-user distance and the antenna intensity (average antenna number per cell). $\alpha=4, K=1, R=1000$.
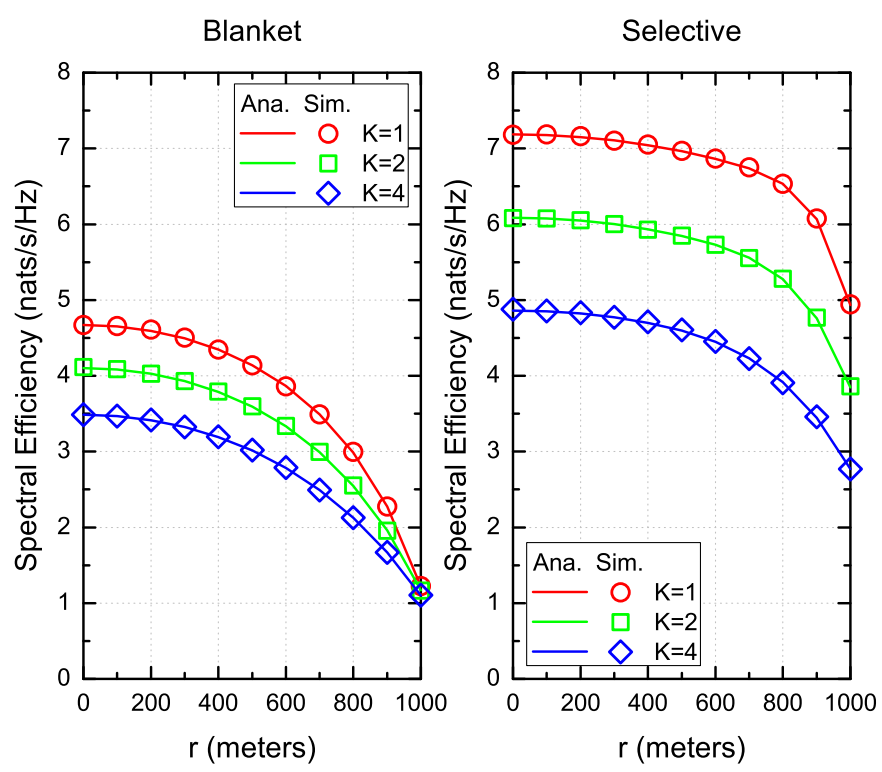

Fig. 5. Regular DAS: spectral efficiency as a function of the cell-center-touser distance and the antenna number per port. $\alpha=4, N=24, R=1000$.

closest serving antenna port, thus improves spectral efficiency. Note that the simulation results with $N=1$ for the blanket and selective cases are not the same. It is because $N$ is the average number of antennas (or antenna ports as $K=1$ ) per cell. When $N=1$ it is also possible that a cell contains multiple antenna ports, all of which are used in blanket transmission while only one is selected in selective transmission.

In Fig. 5, we fix the total average number of the antennas per cell and vary the number of antennas per port (thus the average number of antenna ports per cell is $\frac{N}{K}$ ), and show that the fully distributed case $(K=1)$ outperforms the partially distributed case $(K>1)$. Fig. 6 investigates the effect of the path loss exponent and shows that the largest spectral
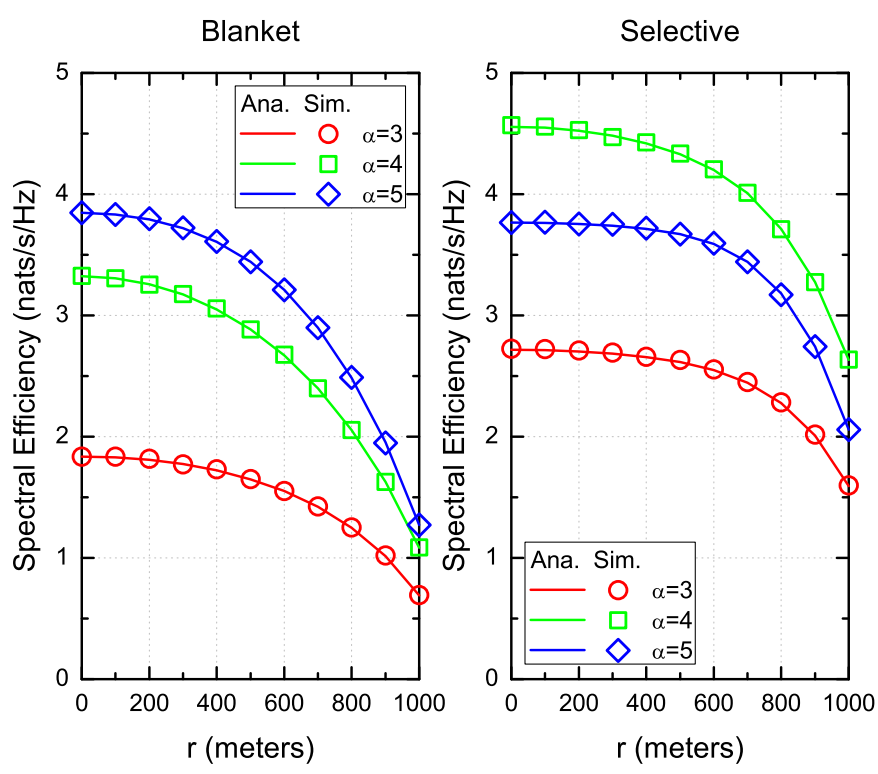

Fig. 6. Regular DAS: spectral efficiency as a function of the cell-center-touser distance and the path loss exponent. $N=6, K=1, R=1000$.
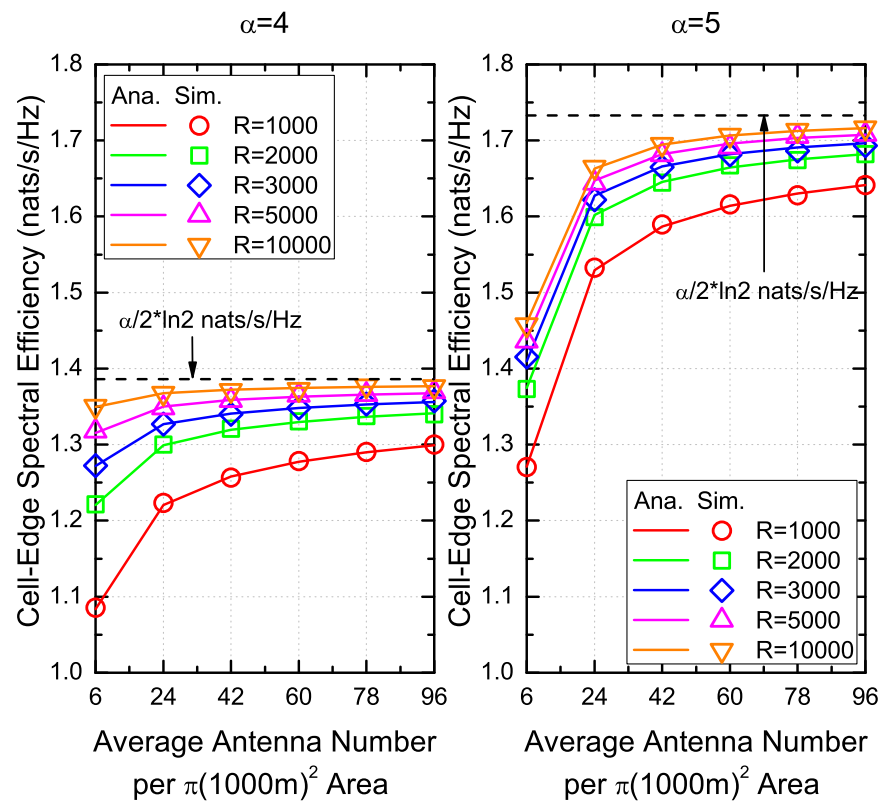

Fig. 7. Regular DAS: cell-edge spectral efficiency as a function of the antenna intensity (average antenna number per $\pi(1000 m)^{2}$ area) and the cell radius. $K=1, r=R$.

efficiency is achieved at $\alpha=5$ for blanket transmission and $\alpha=4$ for selective transmission, respectively.

From Figs. 4-6, we see that selective transmission outperforms blanket transmission in spectral efficiency for most cases at both the cell center and the cell edge, which is in accordance with the conclusion in [4]. This is because in selective transmission only the antennas closest to the active users are transmitting; antenna ports far from the user do not add much signal for the desired user while they contribute to the interference of adjacent cell users ${ }^{1}$. The only exception

${ }^{1}$ We note that it is possible for an intermediate approach, e.g., $k$-selective scheme, to yield better results, but such a scheme is more difficult to analyze. 
occurs at the cell center $(r=0)$ with $\alpha=5$ (Fig. 6) where blanket transmission is slightly better than selective transmission. This happens because at high attenuation the radiation power declines more rapidly with distance. For the user at the cell center area, since interfering antennas are always farther away than serving antennas, under the high attenuation scenario, the interference power declines faster than the desired signal power, so in this case it is beneficial to use all available antenna ports to enhance the desired signals.

Observe that in Figs. 4-5, with the same path loss exponent, the spectral efficiencies of blanket transmission converge at the cell edge to a single point regardless of the antenna intensities. We further confirm the cell-edge spectral efficiency upper bound of blanket transmission in Fig. 7, where spectral efficiency increases with the antenna intensity and the cell radius, but is always upper bounded by $\frac{\alpha}{2} \ln 2$ nats $/ \mathrm{sec} / \mathrm{Hz}$. However, no such upper bound is observed for selective transmission at cell edge in Figs. 4-5. This underscores the limitation of blanket transmission in regular DAS.

\section{Spectral EfFiciency of Regular And User-Centric DAS From a Network Perspective}

In the location-specific analysis of regular DAS, we assume exactly one user is served per cell and focus on the spectral efficiency of such a user at a given location. This singleuser assumption is adopted in many previous works on DAS, e.g., [4], [8], [18], [19], but it does not take the effect of scheduling into account. This section analyzes the average spectral efficiency from a network perspective by considering random distribution of users as well. Specifically, we assume that users are also distributed as a PPP denoted as $\Phi_{U}$ with intensity $\lambda_{U}$, superposed on the antenna PPP $\Phi_{A}$. In this case, a cell may contain no users or more than one user.

Without transmit CSI, if multiple other users are present in the cell, TDMA can be used as a multiple access scheme [15]. In this section, we define the network spectral efficiency as the expected spectral efficiency of a randomly chosen user (termed the typical user) by assuming that TDMA is employed in the cell and every user has an equal probability to be selected for service (or has an equal share of resources). For regular DAS, a crucial difference between the network spectral efficiency and the location-specific spectral efficiency considered earlier is that the network spectral efficiency is not a monotonic function of the cell radius. Intuitively, a larger cell radius allows more antennas to be in the cooperating cluster, but also allows more users in the cluster, which reduces the selection probability of each user. Therefore, from a network spectral efficiency perspective, there exists an optimal cell radius for regular DAS.

We first provide an approximated network spectral efficiency analysis for regular DAS by considering the random user distribution both within and outside of the considered cell. This approximation facilitates performance comparison for both blanket and selective transmission, and indicates the existence of an optimal cell radius from numerical evaluation. We then investigate the network spectral efficiency of a user-centric DAS layout under random user distribution, which is different from the regular DAS layout in that no fixed cell boundaries exist. Finally, the network spectral efficiency comparison of all DAS schemes are given.

\section{A. Regular DAS with Fixed Cell Boundaries}

Under random user distribution, there might be zero or multiple other users, apart from the typical user, in the considered cell. The following lemma deals with the selection probability with multiple users present in the cell.

Lemma 3: With PPP-modeled users, the selection probability of (or the fraction of the resources allocated to) the typical user in a cell with radius $R$, is

$$
\mathbb{P}_{U}^{(\text {Reg. })}=\frac{1-e^{-\pi R^{2} \lambda_{U}}}{\pi R^{2} \lambda_{U}} .
$$

Proof: We add the typical user at the origin of the 2D plane in addition to the user PPP $\Phi_{U}$. With random shift of the regular cellular boundaries on the plane, the relative location of the typical user within its cell is random. According to the Slivnyak's theorem [17], the locations of other users that fall in the same cell of the typical user follow the same original distribution. Thus, the probability mass function of the number of other users (denoted as $M$ ) in the cell of the typical user is Poisson

$$
\mathbb{P}(M=m)=\frac{\left(\pi R^{2} \lambda_{U}\right)^{m}}{m !} e^{-\pi R^{2} \lambda_{U}} .
$$

The selection probability of the typical user is thus

$$
\mathbb{P}_{U}^{(\text {Reg. })}=\sum_{m=0}^{\infty} \frac{\mathbb{P}(M=m)}{m+1} .
$$

By evaluating the above summation we have (28).

We can verify that $\lim _{R \rightarrow 0} \mathbb{P}_{U}=1, \lim _{R \rightarrow \infty} \mathbb{P}_{U}=0$, and that $\mathbb{P}_{U}$ monotonically decreases with $R$.

Since the number of users in neighboring cells can be zero, not all neighboring cells produce interference to the typical cell at each snapshot. Under the regular cellular boundaries, it is not easy to model the spatial distribution of the interferers exactly. In this paper we adopt an approximation by assuming independent thinning of the interference PPP.

For blanket transmission, a neighboring cell does not produce interference if there are no users in that cell. Thus we assume that the intensity of the interfering antenna port is thinned by the probability of non-zero-user event of its cell, i.e., the interference set $\Phi_{A}^{(I)}$ can be approximated as a PPP with intensity $\lambda_{A}^{\left(I^{\prime}\right)}=\left(1-e^{-\pi R^{2} \lambda_{U}}\right) \lambda_{A}$. For selective transmission, if there are either no antennas or no users in a cell, no interference is generated from that cell. Hence the interference set $\Phi_{A}^{(I)}$ can be approximated as a PPP with intensity $\lambda_{A}^{\left(I^{\prime \prime}\right)}=\left(1-e^{-\pi R^{2} \lambda_{A}}\right)\left(1-e^{-\pi R^{2} \lambda_{U}}\right) \frac{1}{\pi R^{2}}$.

Considering the selection probability and the reduced interference intensity, and averaging over all possible user locations within the cell, we have the following proposition for the network spectral efficiency of regular DAS under random user distribution.

Proposition 2: Assuming users are PPP-modeled, the network spectral efficiency of regular DAS averaged over the cell 
is

$$
\mathcal{C}_{\text {Netw. }}^{\text {(Reg. }}=\int_{0}^{R} \mathcal{C}_{r}^{\text {(Reg.) }} f_{r}(r) \mathrm{d} r, \quad \text { where } f_{r}(r)=\frac{2 r}{R^{2}},
$$

where $\mathcal{C}_{r}^{(\text {Reg. })}$ is the location-specific spectral efficiency at a distance $r$ from the cell center of any of the two transmission schemes

$$
\mathcal{C}_{r}^{(\text {Reg. })} \approx \begin{cases}\mathbb{P}_{U}^{(\text {Reg. })} \mathcal{C}_{r}^{(\text {Blk. })}\left(\lambda_{A}^{\left(I^{\prime}\right)}\right) & \text { blanket transmission } \\ \mathbb{P}_{U}^{(\text {Reg. })} \mathcal{C}_{r}^{(\text {Sel. })}\left(\lambda_{A}^{\left(I^{\prime \prime}\right)}\right) & \text { selective transmission. }\end{cases}
$$

$\mathcal{C}_{r}^{(\text {Blk. })}\left(\lambda_{A}^{\left(I^{\prime}\right)}\right)$ and $\mathcal{C}_{r}^{(\text {Sel. })}\left(\lambda_{A}^{\left(I^{\prime \prime}\right)}\right)$ are the location-specific spectral efficiencies (8) and (26), with point processes substituted by PPPs with intensities $\lambda_{A}^{\left(I^{\prime}\right)}$ and $\lambda_{A}^{\left(I^{\prime \prime}\right)}$ in the Laplace transform of the interference parts, respectively. $\mathbb{P}_{U}^{(\text {Reg. })}$ is given in (28).

We demonstrate the effectiveness of the approximations used for the interference intensities $\lambda_{A}^{\left(I^{\prime}\right)}$ and $\lambda_{A}^{\left(I^{\prime \prime}\right)}$ in the simulation part of this paper.

\section{B. User-Centric Selective DAS}

In the previous regular DAS schemes, the entire network is partitioned into cells with fixed boundaries. In this section, we consider a user-centric DAS layout where all regular cell boundaries are removed. In this case, each user finds its closest antenna port as its serving port, which we refer to as the usercentric selective (UC-Sel.) DAS scheme. Note that user-centric selective DAS is different from regular selective DAS, where in the latter scheme the closest antenna port is searched within the cell. Since in DAS networks there are usually more antenna ports than users, i.e., $\lambda_{A}>\lambda_{U}$, this architecture is also known as the ultra-dense network [30].

With the distance based association, the antenna ports form the Voronoi tessellation [28]. Since $\Phi_{U}$ and $\Phi_{A}$ are independent, the Voronoi cell containing the typical user may contain none or multiple other users. In the latter case, TDMA is adopted as a user access scheme. From the Proposition 2 in [6], the selection probability of the typical user is

$$
\mathbb{P}_{U}^{(\text {UC-Sel. })}=\frac{\lambda_{A}}{\lambda_{U}}\left[1-\left(1+3.5^{-1} \frac{\lambda_{U}}{\lambda_{A}}\right)^{-3.5}\right],
$$

which is only a function of the ratio of the antenna port and the user intensity.

Conditioned on the distance between the considered user and its closest antenna port $d$, the Laplace transform of the signal part in (4) is

$$
\mathcal{G}_{\Phi_{A}^{(S)} \mid d}^{(\mathrm{UC}-\mathrm{Sel} .)}=\mathbb{E}_{G}\left(e^{-s G d^{-\alpha}}\right)=\left(1+\mu s d^{-\alpha}\right)^{-K} .
$$

Considering that antenna ports do not generate interference if their Voronoi cells do no contain users, the interfering antenna ports set $\Phi_{A}^{(I)}$ can be approximated as a PPP with intensity [6]

$$
\lambda_{A}^{(\mathrm{UC}-\mathrm{Sel} .)}=\lambda_{A}\left[1-\left(1+3.5^{-1} \frac{\lambda_{U}}{\lambda_{A}}\right)^{-3.5}\right] .
$$

Similar to (13), the Laplace transform of the interference part is approximated as

$$
\begin{aligned}
\mathcal{G}_{\Phi_{A}^{(I)} \mid d}^{\text {(UC-Sel. })} & \approx \exp \left\{\lambda_{A}^{\text {(UC-Sel. })} \int_{0}^{2 \pi} \int_{d}^{\infty}\left[\left(1+\mu s v^{-\alpha}\right)^{-K}-1\right] v \mathrm{~d} v \mathrm{~d} \theta\right\} \\
& =\exp \left[\pi \lambda_{A}^{\text {(UC-Sel. })}(\mu s)^{2 / \alpha} \int_{d^{2}(\mu s)^{-2 / \alpha}}^{\infty} \beta(u) \mathrm{d} u\right],
\end{aligned}
$$

where $v>d$ since the interfering antenna ports are farther than the serving port.

The spectral efficiency of user-centric selective DAS can now be obtained using (4). In addition, the network spectral efficiency should be averaged over the distance $d$, since the border between the signal and interference region is not fixed as in regular DAS with fixed cell boundaries.

Proposition 3: For user-centric DAS with selective transmission, the downlink network spectral efficiency has the following approximation

$$
\begin{aligned}
\mathcal{C}_{\text {Netw. }}^{\text {(UC-Sel. })} & =\mathbb{P}_{U}^{\text {(UC-Sel. })} \mathbb{E}_{d}\left(\mathcal{C}_{d}^{\text {(UC-Sel. })}\right) \\
& =\mathbb{P}_{U}^{\text {(UC-Sel. })} \int_{0}^{\infty} \mathcal{C}_{d}^{\text {(UC-Sel. })} f_{d}(d) \mathrm{d} d
\end{aligned}
$$

where

$$
\mathcal{C}_{d}^{\text {(UC-Sel.) }}=\int_{0}^{\infty} \frac{e^{-s \sigma^{2}}}{s} \mathcal{G}_{\Phi_{A}^{(I)} \mid d}^{\text {(UC-Sel. })}\left(1-\mathcal{G}_{\Phi_{A}^{(S)} \mid d}^{(\text {UC-Sel. })}\right) \mathrm{d} s,
$$

and $\mathbb{P}_{U}^{(\mathrm{UC}-S e l .)}, \mathcal{G}_{\Phi_{A}^{(S)} \mid d}^{(\mathrm{UC}-\mathrm{Sel} .)}$, and $\mathcal{G}_{\Phi_{A}^{(I)} \mid d}^{(\mathrm{UC}-S e l .)}$ are given in (33), (34), and (36), respectively. The distribution of $d$ is given as in [21]

$$
f_{d}(d)=e^{-\pi d^{2} \lambda_{A}} 2 \pi d \lambda_{A} .
$$

\section{Comparison of All Schemes}

In this section, we present simulation results for the network spectral efficiency of the regular and user-centric DAS layouts, and compare all DAS schemes. With the same antenna-port and user density as in the previous location-specific analysis with $R=1000 \mathrm{~m}$ radius, we set $\lambda_{U}=\frac{1}{\pi(1000 m)^{2}}$ and $\lambda_{A}=\frac{N}{K \pi(1000 m)^{2}}$ where $N$ here denotes the average number of antennas over an area of $\pi 1000^{2}$. The power per antenna is again assumed to be $\frac{P}{N}$ where $P=46 \mathrm{dBm}$.

First, for regular DAS with fixed cell boundaries, we plot the network spectral efficiency versus different cell radii $R$ in Figs. 8-9. We observe that the analytical results deviate from simulation for blanket transmission, especially at $\alpha=4$ with a high antenna intensity. This inaccuracy comes from the approximation used for the thinning of the interference, i.e., the approximation of $\lambda_{A}^{\left(I^{\prime}\right)}$, where we assume independent thinning of the PPP. Note that under blanket transmission, excluding the cells that do not generate interference (with no users inside), the set of actual interfering antenna ports appears as a clustered process, i.e., superposition of many (perhaps non-adjacent) clusters/cells of points, which is far from the PPP that we use to approximate the interference set $\Phi_{A}^{\left(I^{\prime}\right)}$. At $\alpha=5$, the gap between the analysis and simulation of blanket transmission becomes smaller because of the higher attenuation of the interference. Also, for selective transmission, 

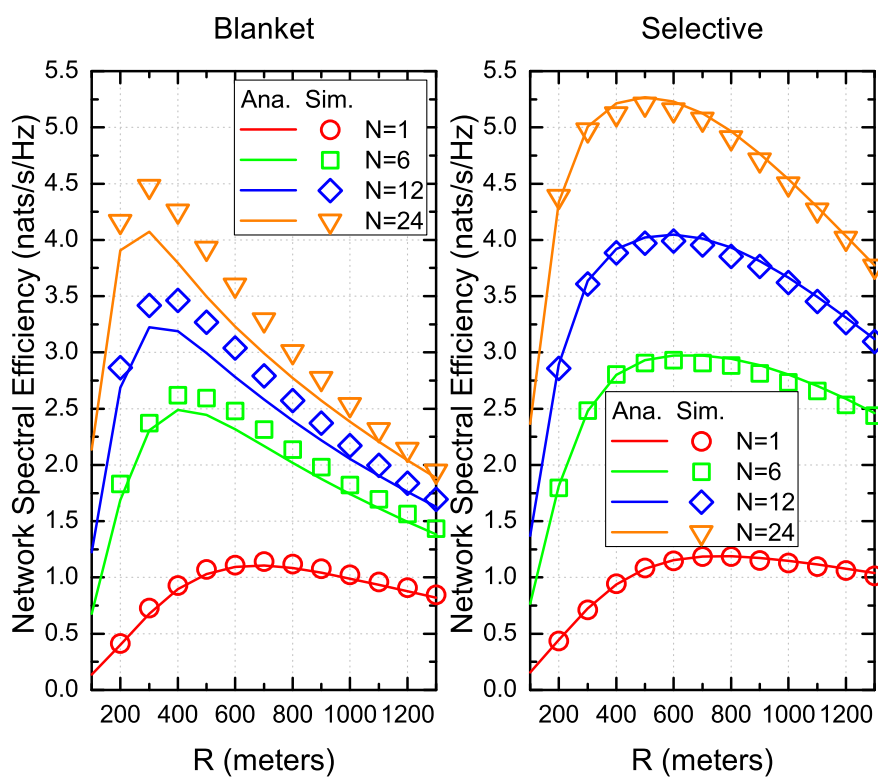

Fig. 8. Network spectral efficiency of regular DAS as a function of the cell radius. $\alpha=4, K=1, \lambda_{U}=\frac{1}{\pi(1000 m)^{2}}$.
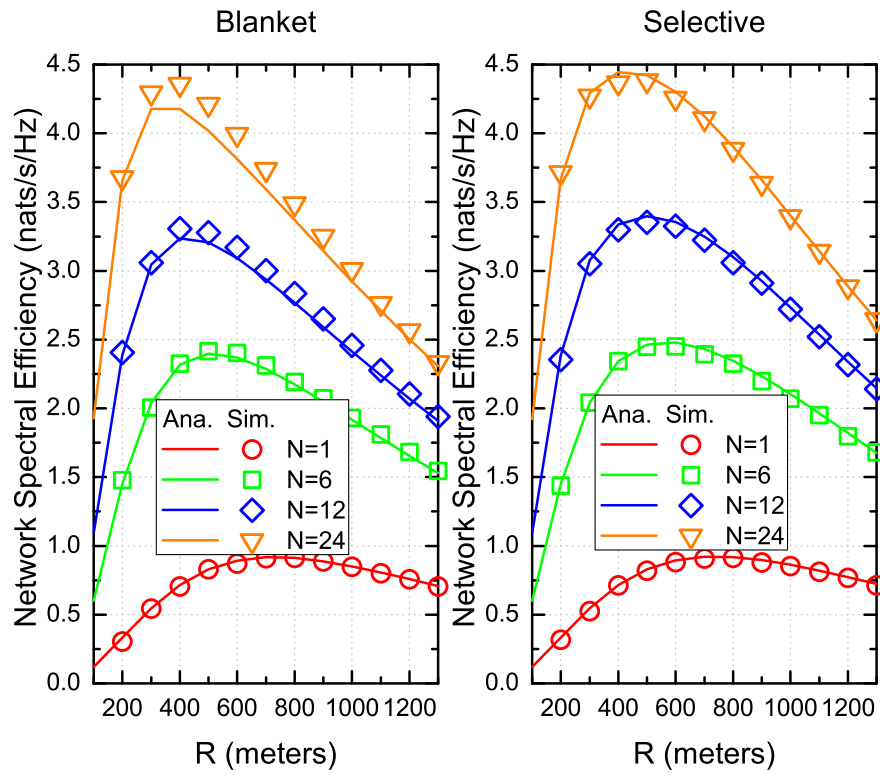

Fig. 9. Network spectral efficiency of regular DAS as a function of the cell radius. $\alpha=5, K=1, \lambda_{U}=\frac{1}{\pi(1000 m)^{2}}$.

the analytical results are always close to simulation. This is because there is always one active antenna port per interfering cell in this case, and the interference set $\Phi_{A}^{\left(I^{\prime \prime}\right)}$ forms a point process (although not Poisson), which is closer to a PPP as compared to the case of a clustered process.

As verified in Figs. 8-9, the network spectral efficiency is not a monotonic function of $R$, and there exists an optimal cell radius that maximizes the network spectral efficiency, e.g., in Fig. 8 with $\alpha=4$ and $\lambda_{A}=\frac{24}{\pi(1000 m)^{2}}, R=300 \mathrm{~m}$ and $R=500 \mathrm{~m}$ are optimal for blanket and selective transmission, respectively. Qualitatively, with larger cell radius the interferers are farther away, but more users are contained in the cell
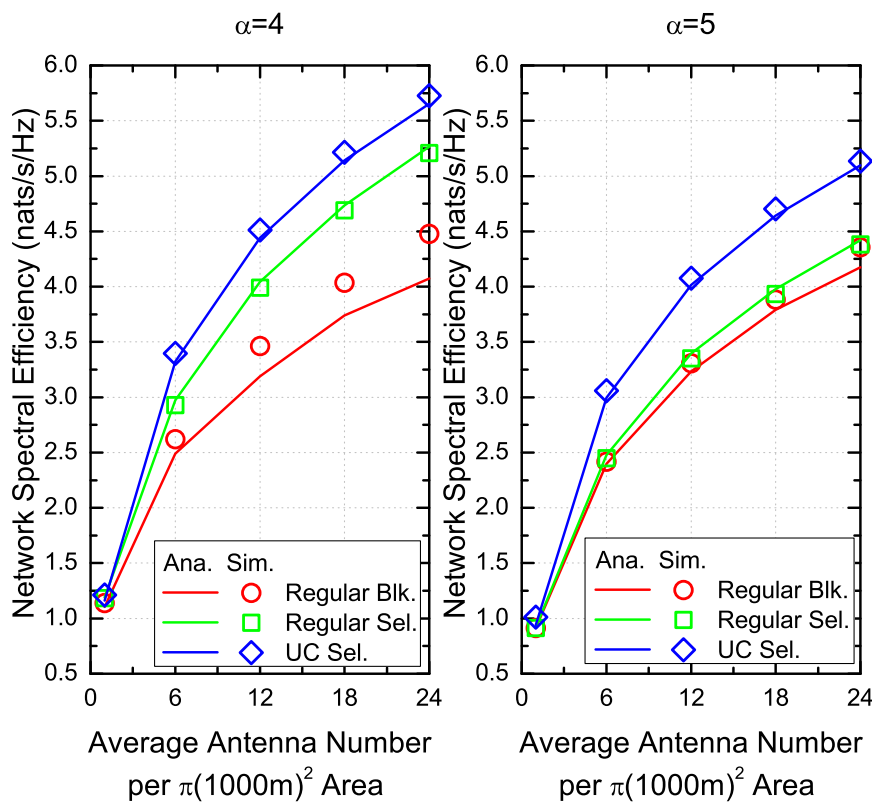

Fig. 10. Network spectral efficiencies of all schemes as a function of the antenna intensity (average antenna number per $\pi(1000 m)^{2}$ area). Regular DAS is plotted with the optimal cell radius. $K=1, \lambda_{U}=\frac{1}{\pi(1000 m)^{2}}$.

and hence the selection probability of the typical user is lower. The analytical results, despite its gap from the simulation, can predict the trend of the network spectral efficiency as a function of the cell radius, and is useful in determining the optimal radius. With the optimal radius, the network spectral efficiency of selective transmission outperforms that of blanket transmission at $\alpha=4$; while the two schemes have similar performance at $\alpha=5$. The optimal radius of selective transmission is greater than that of blanket transmission under the same antenna intensity; for both transmission schemes it decreases with the antenna port intensity.

In Fig. 10, we compare regular DAS and user-centric selective DAS as the antenna port intensity varies. The regular DAS schemes are plotted with its optimal cell radius as determined from Figs. 8-9. Compared to regular selective DAS with circular cell boundaries, user-centric selective DAS has better performance. One reason for this is that, the cell size of regular selective DAS with the optimal radius is still on average larger than that of the Voronoi cells of user-centric selective DAS, thus a regular cell contains more users which reduces the user selection probability. This loss in available transmission opportunity is not compensated by the interference reduction due to the interferers being pushed away with a larger cell size. Further, under the PPP model: 1) the selected antenna port of regular DAS may not be the closest to the user, since such selection is not done over the whole network but within one cell; 2) with fixed cell boundaries, there is a nonzero probability $\mathbb{P}_{A}(\overline{\text { void }})$ that there are no antennas in the cell. These factors explain the inferior performance of regular selective DAS as compared to user-centric selective DAS.

Finally we comment that since each antenna port acts as an independent access point like a micro base-station, employing user-centric selective DAS is in principle equivalent to 
splitting cells into smaller cells hence densifying the network. This may add to the architecture complexity of the network since the number of independent control units need to grow proportionally.

\section{CONCLUSION}

In this paper, we study random antenna distribution and PPP based modeling of DAS. Assuming no transmit CSI and per-antenna power constraint, we derive the downlink spectral efficiency of DAS in a tractable form for efficient numerical computation. We present the spectral efficiency results of DAS with either blanket or selective transmission under fixed cell boundaries, as well as user-centric selective DAS where cell boundaries are removed. The proposed analytical results facilitate the comparison of different DAS schemes, and their effectiveness is demonstrated by numerical simulation. For regular DAS with cell boundaries, we show that it has better cell-edge spectral efficiency than the traditional cellular system, and the selective scheme outperforms the blanket scheme. From a network perspective where users are also randomly distributed and assuming a TDMA-based user access, usercentric selective DAS is shown to outperform both regular DAS schemes.

\section{REFERENCES}

[1] A. Saleh, A. Rustako, and R. Roman, "Distributed antennas for indoor radio communications," IEEE Trans. Commun., vol. 35, no. 12, pp. 1245-1251, Dec. 1987.

[2] K. Kerpez, "A radio access system with distributed antennas," IEEE Trans. Veh. Technol., vol. 45, no. 2, pp. 265-275, May 1996.

[3] S. Zhou, M. Zhao, X. Xu, J. Wang, and Y. Yao, "Distributed wireless communication system: a new architecture for future public wireless access," IEEE Commun. Magazine, vol. 41, no. 3, pp. 108-113, Mar. 2003.

[4] W. Choi and J. Andrews, "Downlink performance and capacity of distributed antenna systems in a multicell environment," IEEE Trans. Wireless Commun., vol. 6, no. 1, pp. 69-73, Jan. 2007.

[5] L. Dai, S. Zhou, and Y. Yao, "Capacity analysis in CDMA distributed antenna systems," IEEE Trans. Wireless Commun., vol. 4, no. 6, pp. 2613-2620, Nov. 2005.

[6] S. M. Yu and S.-L. Kim, "Downlink capacity and base station density in cellular networks," in Proc. IEEE WiOpt Workshop on Spatial Stochastic Models for Wireless Networks (SpaSWiN), May 2013.

[7] J. Park, E. Song, and W. Sung, "Capacity analysis for distributed antenna systems using cooperative transmission schemes in fading channels," IEEE Trans. Wireless Commun., vol. 8, no. 2, pp. 586-592, Feb. 2009.

[8] S. Firouzabadi and A. Goldsmith, "Downlink performance and capacity of distributed antenna systems." [Online]. Available: arXiv:1109.2957

[9] R. Heath, T. Wu, Y. Kwon, and A. Soong, "Multiuser MIMO in distributed antenna systems with out-of-cell interference," IEEE Trans. Signal Process., vol. 59, no. 10, pp. 4885-4899, Oct. 2011.

[10] H. Zhuang, L. Dai, L. Xiao, and Y. Yao, "Spectral efficiency of distributed antenna system with random antenna layout," Electronics Letters, vol. 39, no. 6, pp. 495-496, Mar. 2003.

[11] J. Zhang and J. Andrews, "Distributed antenna systems with randomness," IEEE Trans. Wireless Commun., vol. 7, no. 9, pp. 3636-3646, Sept. 2008.

[12] W. Feng, Y. Li, S. Zhou, J. Wang, and M. Xia, "Downlink capacity of distributed antenna systems in a multi-cell environment," in Proc. IEEE Wireless Commun. Netw. Conf. (WCNC), Apr. 2009, pp. 1-5.

[13] W. Feng, Y. Li, J. Gan, S. Zhou, J. Wang, and M. Xia, "On the deployment of antenna elements in generalized multi-user distributed antenna systems," Mobile Netw. Appl., vol. 16, no. 1, pp. 35-45, Feb. 2011.

[14] L. Dai, "A comparative study on uplink sum capacity with co-located and distributed antennas," IEEE J. Sel. Areas Commun., vol. 29, no. 6, pp. 1200-1213, June 2011.
[15] D. Wang, J. Wang, X. You, Y. Wang, M. Chen, and X. Hou, "Spectral efficiency of distributed MIMO systems," IEEE J. Sel. Areas Commun., vol. 31, no. 10, pp. 2112-2127, Oct. 2013.

[16] M. Haenggi, J. Andrews, F. Baccelli, O. Dousse, and M. Franceschetti, "Stochastic geometry and random graphs for the analysis and design of wireless networks," IEEE J. Sel. Areas Commun., vol. 27, no. 7, pp. 1029-1046, Sept. 2009.

[17] M. Haenggi, Stochastic Geometry for Wireless Networks. Cambridge University Press, 2012.

[18] S.-R. Lee, S.-H. Moon, J.-S. Kim, and I. Lee, "Capacity analysis of distributed antenna systems in a composite fading channel," IEEE Trans. Wireless Commun., vol. 11, no. 3, pp. 1076-1086, Mar. 2012.

[19] F. Héliot, R. Hoshyar, and R. Tafazolli, "An accurate closed-form approximation of the distributed mimo outage probability," IEEE Trans. Wireless Commun., vol. 10, no. 1, pp. 5-11, Jan. 2011.

[20] E. Park, S.-R. Lee, and I. Lee, "Antenna placement optimization for distributed antenna systems," IEEE Trans. Wireless Commun., vol. 11, no. 7, pp. 2468-2477, July 2012.

[21] J. Andrews, F. Baccelli, and R. Ganti, "A tractable approach to coverage and rate in cellular networks," IEEE Trans. Commun., vol. 59, no. 11 , pp. 3122-3134, Nov. 2011.

[22] T. Novlan, R. Ganti, A. Ghosh, and J. Andrews, "Analytical evaluation of fractional frequency reuse for OFDMA cellular networks," IEEE Trans. Wireless Commun., vol. 10, no. 12, pp. 4294-4305, Dec. 2011.

[23] H. Jo, Y. Sang, P. Xia, and J. Andrews, "Heterogeneous cellular networks with flexible cell association: A comprehensive downlink SINR analysis," IEEE Trans. Wireless Commun., vol. 11, no. 10, pp. 34843495, Oct. 2012.

[24] H. Dhillon, R. Ganti, F. Baccelli, and J. Andrews, "Modeling and analysis of $K$-tier downlink heterogeneous cellular networks," IEEE J. Sel. Areas Commun., vol. 30, no. 3, pp. 550-560, Apr. 2012.

[25] S. Singh, H. Dhillon, and J. Andrews, "Offloading in heterogeneous networks: Modeling, analysis, and design insights," IEEE Trans. Wireless Commun., vol. 12, no. 5, pp. 2484-2497, May 2013.

[26] M. Vu, "MISO capacity with per-antenna power constraint," IEEE Trans. Commun., vol. 59, no. 5, pp. 1268-1274, May 2011.

[27] K. Hamdi, "Capacity of MRC on correlated Rician fading channels," IEEE Trans. Commun., vol. 56, no. 5, pp. 708-711, May 2008

[28] A. Okabe, B. Boots, K. Sugihara, and S. N. Chiu, Spatial tessellations: concepts and applications of Voronoi diagrams. Wiley, 2009.

[29] R. Heath and M. Kountouris, "Modeling heterogeneous network interference," in Proc. Inf. Theory App. Workshop (ITA), Feb. 2012, pp. 17-22.

[30] I. Hwang, B. Song, and S. S. Soliman, "A holistic view on hyperdense heterogeneous and small cell networks," IEEE Communications Magazine, vol. 51, no. 6, pp. 20-27, June 2013. 